\title{
MODELING THE OVERSHOOTING EFFECT OF MULTI-INPUT GATE IN NANOMETER TECHNOLOGIES*
}

\author{
LI DING \\ Graduate School of Information, Production and Systems, Waseda University, \\ 2-7 Hibikino, Wakamatsu-Ku, Kitakyushu-Shi, 808-0135, Japan \\ dingli@fuji.waseda.jp \\ ZHANGCAI HUANG \\ Information, Production and Systems Research Center, Waseda University, \\ 2-7 Hibikino, Wakamatsu-Ku, Kitakyushu-Shi, 808-0135, Japan \\ hzc_2002@asagi.waseda.jp \\ MINGLU JIANG \\ Graduate School of Information, Production and Systems, Waseda University, \\ 2-7 Hibikino, Wakamatsu-Ku, Kitakyushu-Shi, 808-0135, Japan \\ jiangminglv1983@fuji.waseda.jp \\ ATSUSHI KUROKAWA \\ Graduate School of Science and Technology, Hirosaki University, \\ 3-bunkyocho, Hirosaki-shi, Aomori-ken 036-8561, Japan \\ kurokawa@eit.hirosaki-u.ac.jp \\ YASUAKI INOUE \\ Graduate School of Information, Production and Systems, Waseda University, \\ 2-7 Hibikino, Wakamatsu-Ku, Kitakyushu-Shi, 808-0135, Japan \\ inoue_yasuaki@waseda.jp \\ Received 31 October 2011 \\ Accepted 8 March 2012 \\ Published 12 November 2012
}

\begin{abstract}
With the advent of nanometer age in digital circuits, the overshooting time becomes an important component of gate delay for CMOS logic gates. However, there has been little attention paid to the research of the overshooting effect for multi-input gate in nanometer technologies until now. Therefore, in this paper, an effective model considering the overshooting
\end{abstract}

*This work was partly presented at the 54th IEEE International Midwest Symposium on Circuits and Systems, Seoul, Korea, 8-10 August, 2011. This paper was recommended by Regional Editor Majid Ahmadi. 
effect of multi-input gate is presented. The experimental results using 32-nm PTM model reflect that the proposed model is accurate within $3.6 \%$ error compared with SPICE simulation results.

Keywords: Nanometer age; multi-input gate; overshooting effect; gate delay.

\section{Introduction}

With digital integrated circuits entering nanometer age, the overshooting effect which is induced by input-to-output coupling capacitance strongly affects CMOS gate delay analysis, especially the static timing analysis. For traditional long channel transistors, the overshooting time is generally ignored because the coupling capacitance is small and the overshooting effect can usually be neglected. ${ }^{1}$ However, with the scaling of gate feature sizes, the overshooting time is becoming equal to gate delay or even larger than that. ${ }^{2}$ Therefore, the overshooting effect should be specially considered during the analysis of gate delay, otherwise the expression of gate delay would be inaccurate.

Traditional models of gate delay have been widely studied. Alpha-power law MOSFET model is applied to derive closed-form equation for evaluating the propagation delay in Ref. 3. An extension to the delay expression of Ref. 3 is presented in Ref. 4 to deal with the case of very lightly loaded inverter and/or slow input signals. However, neither Ref. 3 nor Ref. 4 takes consideration of the overshooting effect when analyzing gate delay. In the submicron range, the second-order effect which includes input slope and input-to-output coupling effect becomes the dominant factor. Although the overshooting effect which is induced by the input-to-output coupling effect is taken into account in the form of constant value or very simple expression in Refs. 5-9, there still have been few researchers focusing on the analysis of the overshooting effect in the level of nanometer technologies.

A detailed expression of the overshooting time for an inverter in nanometer technology is obtained by complete calculation and reasonable approximation in Refs. 2 and 10. However, the overshooting effect of multi-input gate is still not considered too much. Therefore, that effect is analyzed specifically in this paper. A basic two-input CMOS NOR gate is chosen as the research object and a practical model of CMOS NOR gate considering the overshooting effect is proposed. The specific expression of the overshooting time for the CMOS NOR gate is obtained from some reasonable calculation and approximation. Moreover, the proposed model is verified by comparing with SPICE simulation results using CMOS 32-nm PTM model. ${ }^{11}$ The simulation results turn out that the accuracy of the proposed model is within $3.6 \%$ error. Furthermore, the proposed model can be easily extended to CMOS NAND gate according to the same principles.

The rest of this paper is composed of four sections as follows. First, the importance of the overshooting time is clarified in Sec. 2. Then the model of the overshooting effect for two-input NOR gate is proposed specifically in Sec. 3. After that, the 
simulation results of the proposed model are shown in Sec. 4. At last the paper is concluded in Sec. 5.

\section{Preliminaries}

The overshooting time $t_{\mathrm{ov}}$ is now a key component of multi-input gate delay in nanometer regime with the scaling of process technology. Figure 1 shows a two-input CMOS NOR gate which takes into account gate-to-drain coupling capacitance and gate-to-source coupling capacitance. Figure 2 shows the output voltage $V_{\text {out }}$ of the two-input NOR gate in the case that $V_{\text {in2 }}$ is connected to a falling signal and $V_{\text {in1 }}$ is directly connected to the ground by using CMOS 32-nm PTM model. ${ }^{11}$ In Fig. 2, $t_{D}$ is the gate delay time defined as the time elapses from $V_{\mathrm{in} 2}=0.5 V_{\mathrm{DD}}$ to $V_{\text {out }}=0.5 V_{\mathrm{DD}}, t_{\mathrm{ov}}$ is the overshooting time while the output voltage is below zero, $t_{r}$ is the output voltage rise time from $t=t_{\mathrm{ov}}$ to the time when $V_{\text {out }}=0.5 V_{\mathrm{DD}}, t_{\text {in }}$ is the transition time of input signal $V_{\mathrm{in} 2}$. Thus, the delay time $t_{D}$ can be expressed as

$$
t_{D}=t_{\mathrm{ov}}+t_{r}-\frac{t_{\text {in }}}{2} .
$$

Figure 3 shows the overshooting time $t_{\mathrm{ov}}$ and the gate delay time $t_{D}$ of the twoinput NOR gate in Fig. 1 for various nanometer process technologies from $90-\mathrm{nm}$ technology to $22-\mathrm{nm}$ technology. The input condition is that $V_{\mathrm{in} 2}$ is connected to a falling signal and $V_{\mathrm{in} 1}$ is connected to the ground. The transistor length $L_{n}$ and $L_{p}$ are the minimum feature size. The PMOS width is $W_{P}=10 L_{P}$ and the NMOS width is $W_{N}=2 W_{P}$. The load capacitance is $C_{L}=0.01 \mathrm{pF}$. The input transition time $t_{\text {in }}$ of $V_{\text {in2 }}$ is 60 ps. In Fig. 3 , both the overshooting time $t_{\text {ov }}$ and the gate delay time $t_{D}$ decrease with the scaling of process technology. On the contrary, the ratio value $t_{\mathrm{ov}} / t_{D}$ increases from $134.7 \%$ in $90 \mathrm{~nm}$ to $249.0 \%$ in $22 \mathrm{~nm}$. Additionally, although $t_{\mathrm{ov}} / t_{D}$ will become small with the decrease of $t_{\text {in }}$, the overshooting time $t_{\mathrm{ov}}$ still

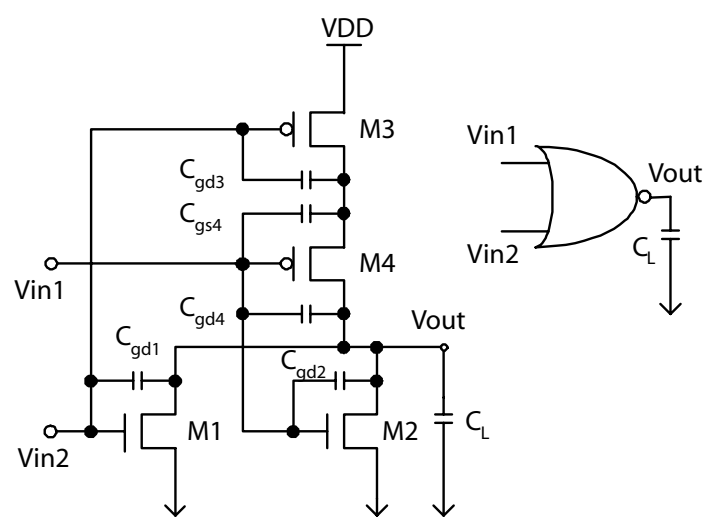

Fig. 1. CMOS NOR gate considering parastical coupling capacitance. 


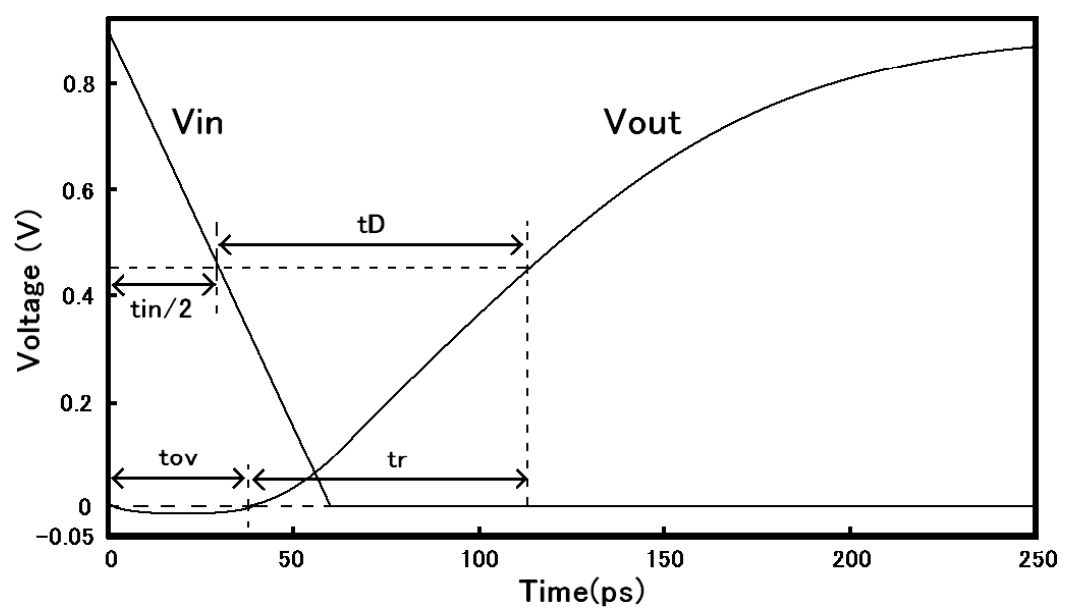

Fig. 2. Output voltage of CMOS NOR gate.

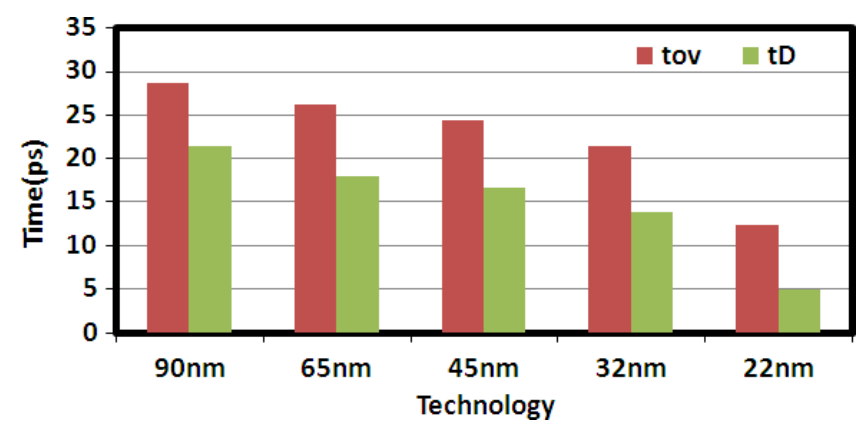

Fig. 3. Overshooting time $t_{\mathrm{ov}}$ and gate delay $t_{D}$ with various process technologies.

occupies a large proportion of delay time $t_{D}$. Therefore, the overshooting effect of CMOS gate should be paid much more attention in nanometer regime. Modeling the overshooting effect of CMOS gate accurately can improve the accuracy of gate delay calculation.

\section{Modeling the Overshooting Effect}

In order to simplify the analysis of modeling the overshooting effect of CMOS NOR gate in Fig. 1, we discuss two different input conditions separately. The first input condition is that the falling signal is applied to $V_{\mathrm{in} 1}$ and $V_{\mathrm{in} 2}$ is connected to the ground. The second input condition is that $V_{\mathrm{in} 1}=0$ and the falling signal is on terminal $V_{\text {in2 }}$. Similar expressions can be obtained if the falling signals are changed to rising signals. 


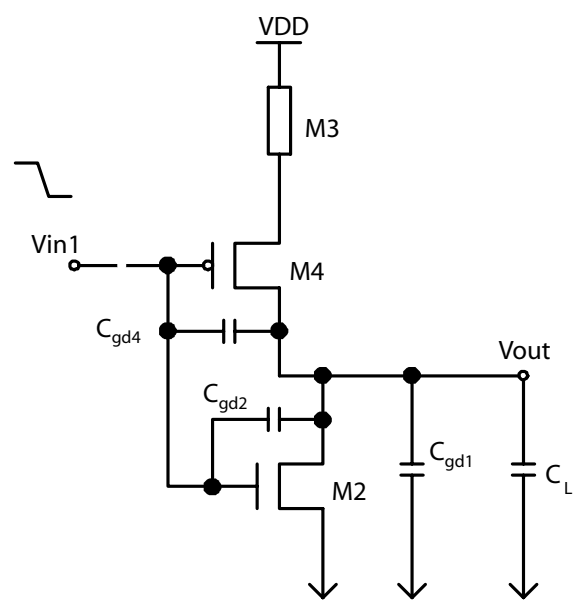

Fig. 4. CMOS NOR gate under the condition $V_{\text {in1 }}$ : High-Low $V_{\text {in2 }}$ : GND.

First, the analysis is given to the case as shown in Fig. 4, where the transistor M1 is not considered because the voltage of its four terminals are almost zero in the overshooting period. At the same time, M3 operates almost in the triode region, and is regarded as an active resistance during the overshooting effect period. The coupling capacitance $C_{g d 3}$ is not considered because the voltage between two nodes of the capacitance is almost constant during the overshooting effect period. Thus, the analysis for NOR is just similar to that for an inverter.

For the simplified NOR gate as shown in Fig. 4 , the $\alpha$-power MOSFET model ${ }^{3}$ is applied to calculate the current of the PMOS transistor. In order to simplify the numerical procedures, the drain current $I_{P}$ of M4 is expressed with linear approximation as shown below:

$$
I_{M 4}\left(t_{\mathrm{ov}}\right)=I_{D 0}\left(\frac{\left|V_{\mathrm{GS}}\right|-\left|V_{\mathrm{TPL}}\right|}{V_{\mathrm{DD}}-\left|V_{\mathrm{TPL}}\right|}\right),
$$

where $I_{D 0}$ is the drain current of a PMOS in saturation at $V_{\mathrm{GS}}=V_{\mathrm{DS}}=V_{\mathrm{DD}}\left(V_{\mathrm{GS}}\right.$ and $V_{\mathrm{DS}}$ are the gate-source and drain-source voltages of the PMOS, respectively), $V_{\mathrm{TPL}}$ is the equivalent threshold voltage whose value is shown in Fig. 5. Actually it is shown in Fig. 5 that the linear approximation about drain current of M4 is almost identical as SPICE simulation result.

Therefore, the specific expression of the overshooting time $t_{\mathrm{ov}}$ is obtained using the method in Ref. 10. First, an initial expression of the overshooting time $t_{\mathrm{ov}}$ is calculated using the linear approximation of drain current for M4. The initial $t_{\mathrm{ov}}$ is expressed as $^{10}$

$$
t_{\mathrm{ov} 1}=t_{V \min }-t_{\gamma}+\sqrt{\frac{2 Q_{1}}{I_{D 0}}\left(t_{\mathrm{in}}-t_{\mathrm{TPL}}\right)+t_{\gamma}^{2}}
$$



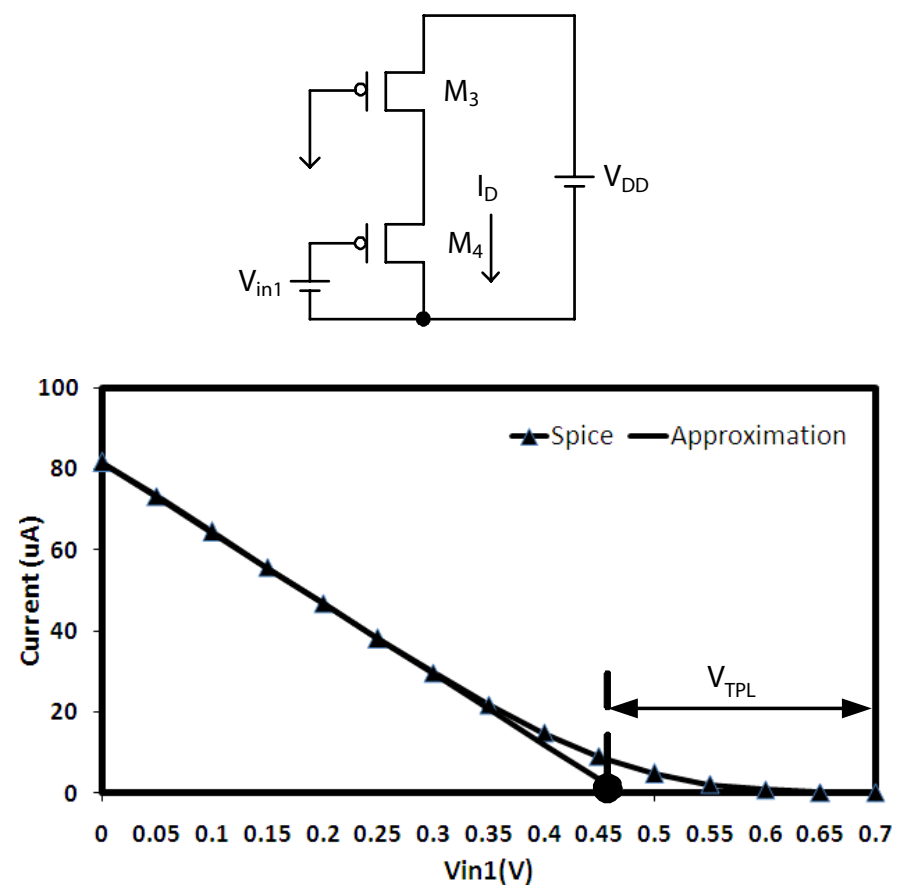

Fig. 5. The linear approximation for PMOS transistor drain current.

where

$$
t_{\gamma}=\frac{1}{2}\left[t_{V \min }-t_{\mathrm{TPL}}-C_{M} \frac{V_{\mathrm{DD}}}{t_{\mathrm{in}}} \frac{t_{\mathrm{in}}-t_{\mathrm{TPL}}}{I_{D 0}}\right]
$$

In Eqs. (3) and (4), $t_{V \min }$ is the time when the output voltage is at its minimum. $t_{\mathrm{TPL}}=\frac{\left|V_{\mathrm{TPL}}\right|}{V_{\mathrm{DD}}} t_{\mathrm{in}}$. Also, $Q_{1}$ is the charge flowing out of the load capacitance $C_{L}$ and $C_{g d 1}$. In this paper, the values of $t_{V \text { min }}$ and $Q_{1}$ is obtained using the method similar to Ref. 10. Moreover, $C_{M}$ is the coupling capacitance and in this paper $C_{M}$ is expressed as

$$
C_{M}=C_{g d 2}+C_{g d 4}
$$

The initial value of the overshooting time in the expression (3) is not accurate enough. Therefore, the initial $t_{\mathrm{ov} 1}$ is substituted into the $\alpha$-power MOSFET model to get a new drain current of M4 $I_{P}\left(t_{\mathrm{ov}}\right)$. Then the final overshooting time for the first input condition is expressed as

$$
t_{\mathrm{ov} 1}=\frac{2 Q_{1}}{C_{M} \frac{V_{\mathrm{DD}}}{t_{\mathrm{in}}}+I_{P}\left(t_{\mathrm{ov}}\right)}+t_{V \min } .
$$




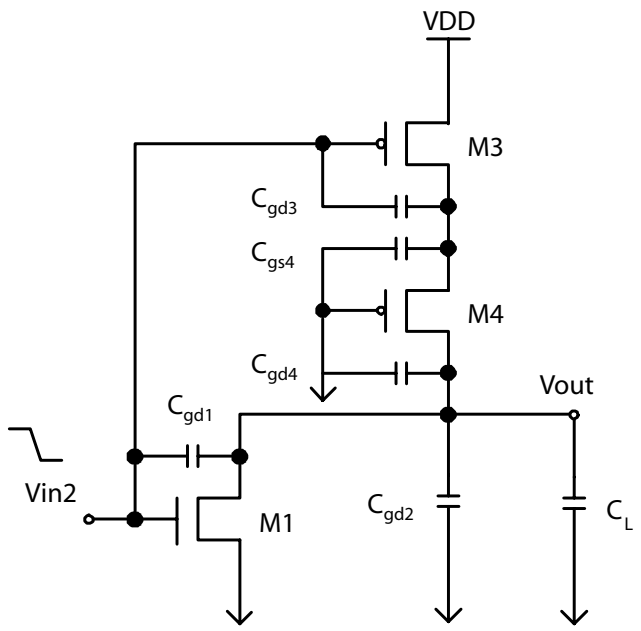

Fig. 6. CMOS NOR gate under the condition $V_{\text {in } 1}$ : GND $V_{\text {in2 }}$ : High-Low.

For the other condition as shown in Fig. 6, the analysis becomes more complicated because the influence of the coupling capacitance $C_{g s 4}$ should also be considered. However, from $t=t_{\mathrm{ov}}$ to $t=t_{r}, \mathrm{M} 4$ operates almost in the triode region, and is regarded as an active resistance. Then the NOR gate in Fig. 6 is simplified as shown in Fig. 7. Through various simulations in different conditions, we find that the overshooting time $t_{\mathrm{ov} 2}$ of the simplified NOR gate in Fig. 7 is proportional to the overshooting time $t_{\mathrm{ov} 1}$ of the simplified inverter in Fig. 4 as shown in Fig. 8. Thus, we

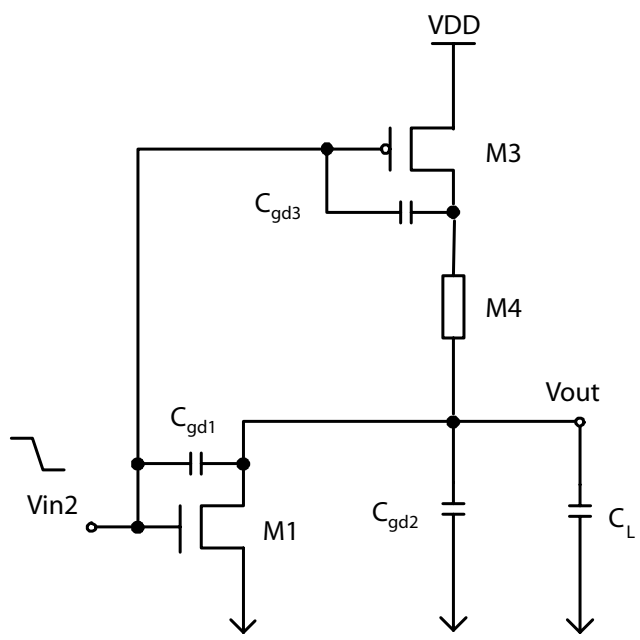

Fig. 7. Simplified CMOS NOR gate under the condition $V_{\text {in1 }}$ : GND $V_{\text {in2 }}$ : High-Low. 


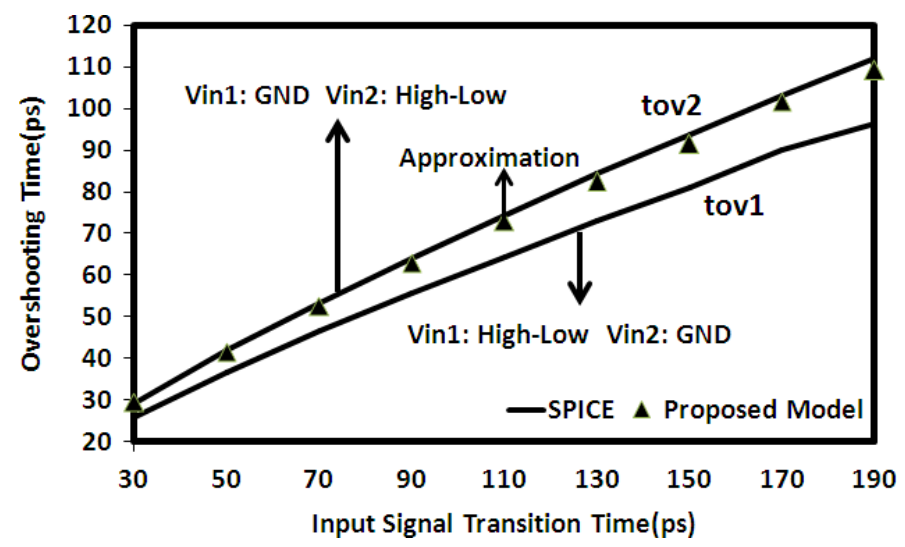

Fig. 8. The linear approximation for the overshooting time.

can obtain $t_{\mathrm{ov} 2}$ referring to the value of $t_{\mathrm{ov} 1}$ as shown below:

$$
t_{\mathrm{ov} 2}=\lambda t_{\mathrm{ov} 1} \text {. }
$$

Figures 9-11 are the simulation verifications of the expression (3) under different input signal transition times, transistor sizes and load capacitances. All these results are obtained from SPICE simulations. The length of NMOS and PMOS are $L_{N}=L_{P}=40 \mathrm{~nm}$.

Figure 9 show the ratio of $t_{\text {ov2 }}$ to $t_{\text {ov1 }}$ under different input signal transition times. The load capacitance is $C_{L}=0.01 \mathrm{pF}$ and the transistor size is $W_{P} / W_{N}=0.8 \mathrm{um} /$ $0.4 \mathrm{um}$. Figure 10 show the ratio of $t_{\mathrm{ov} 2}$ to $t_{\mathrm{ov} 1}$ under different NMOS widths. The PMOS' width is two times of NMOS' width. The input signal transition time is $t_{\text {in }}=100 \mathrm{ps}$ and the load capacitance is $C_{L}=0.02 \mathrm{pF}$. Figure 11 show the ratio of $t_{\text {ov2 }}$ to $t_{\mathrm{ov} 1}$ under different load capacitances. The input signal transition time is $t_{\text {in }}=140 \mathrm{ps}$ and the transistor size is $W_{P} / W_{N}=2.4 \mu \mathrm{m} / 1.2 \mu \mathrm{m}$. Through the

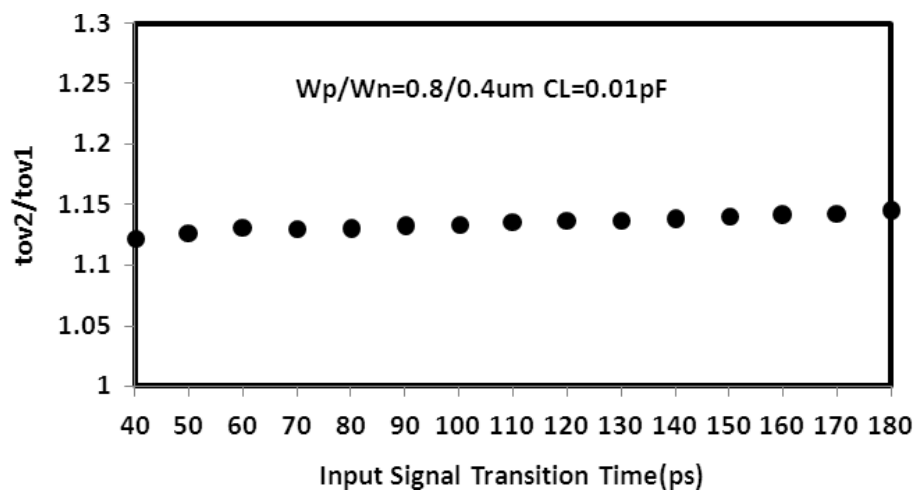

Fig. 9. $t_{\mathrm{ov} 2} / t_{\mathrm{ov} 1}$ versus different input signal transition time. 


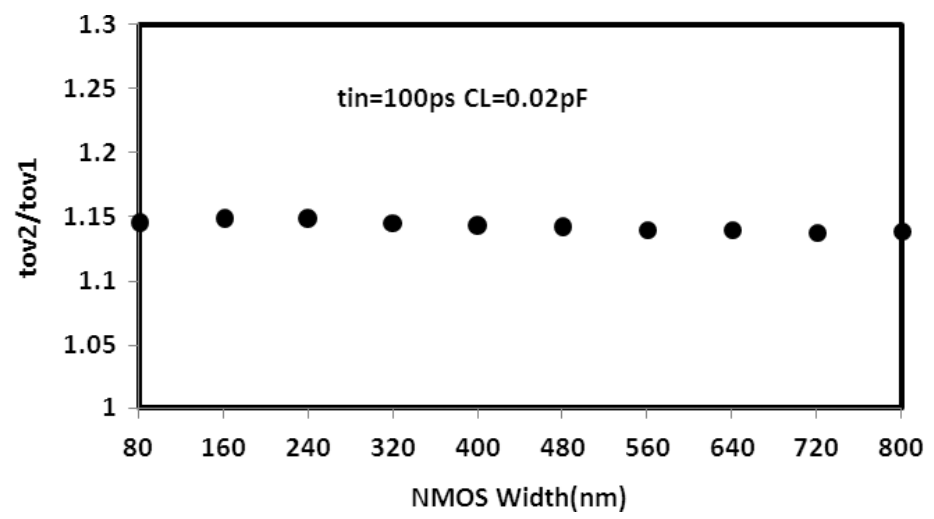

Fig. 10. $t_{\mathrm{ov} 2} / t_{\mathrm{ov} 1}$ versus different gate sizes.

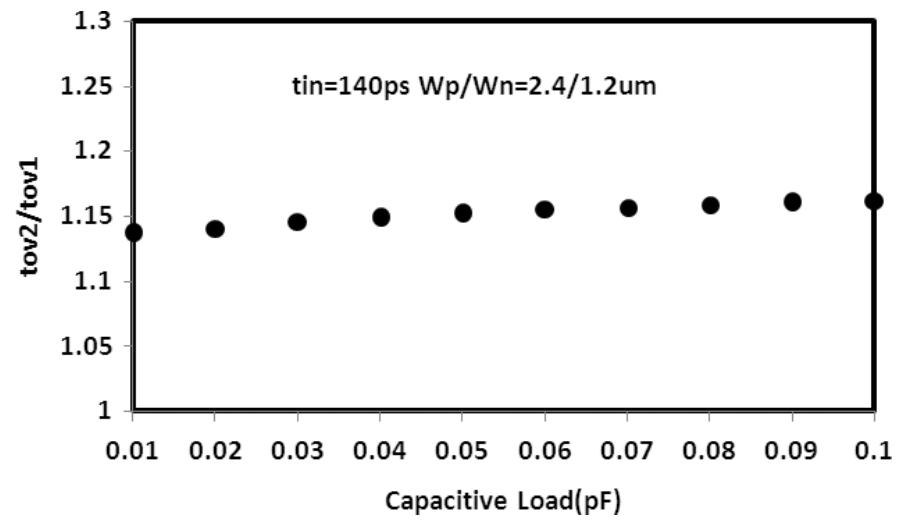

Fig. 11. $t_{\mathrm{ov} 2} / t_{\mathrm{ov} 1}$ versus different capacitive loads.

verification as shown in Figs. 9-11, we can find that the ratio is almost constant and the value is approximately equal to 1.14 . Therefore $\lambda$ is about 1.14 for the $32-\mathrm{nm}$ PTM model. ${ }^{11}$ Based on other numerous simulations for various conditions, it is demonstrated that $\lambda$ makes little change with the variation of input signal transition time, transistor size or capacitive load. Additionally, $\lambda$ for different process technology can be obtained in the similar way.

\section{Simulation Results of the Overshooting Time}

Figures 12-14 show the overshooting time obtained from the proposed model and SPICE simulations for the first input condition with respect to various input signal transition times, transistor sizes and load capacitances using 32-nm PTM model. ${ }^{11}$ Here the input signal transition time $t_{\text {in }}$ varies from 40 to $120 \mathrm{ps}$. The NMOS' width 
L. Ding et al.

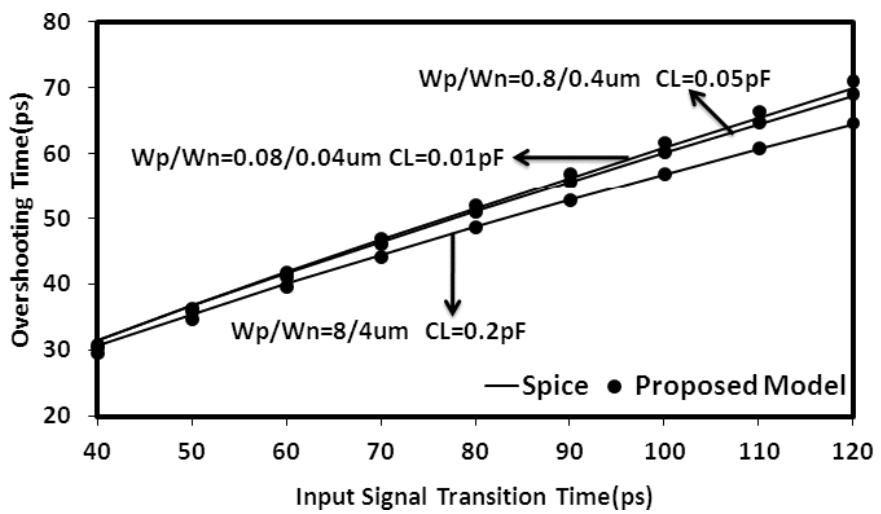

Fig. 12. The overshooting time $t_{\mathrm{ov}}$ of the proposed model and SPICE simulation for CMOS NOR gate (Fig. 4) with respect to various input signal transition times.

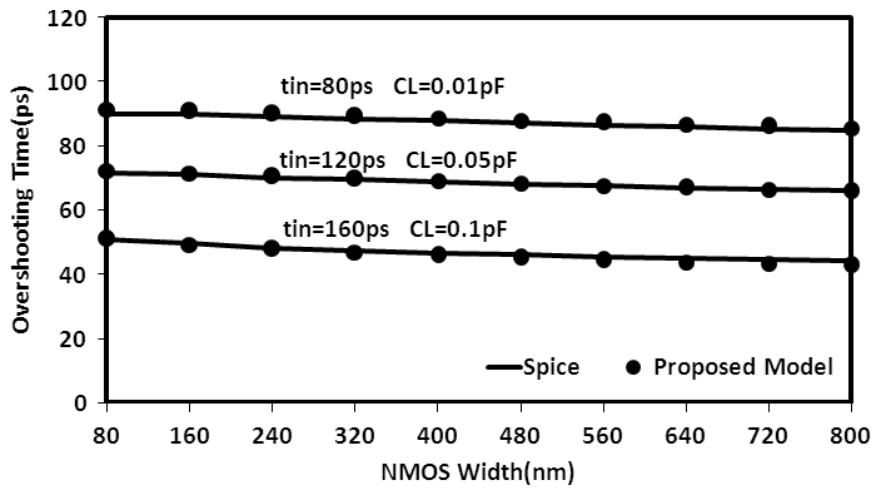

Fig. 13. The overshooting time $t_{\mathrm{ov}}$ of the proposed model and SPICE simulation for CMOS NOR gate (Fig. 4) with respect to various gate sizes.

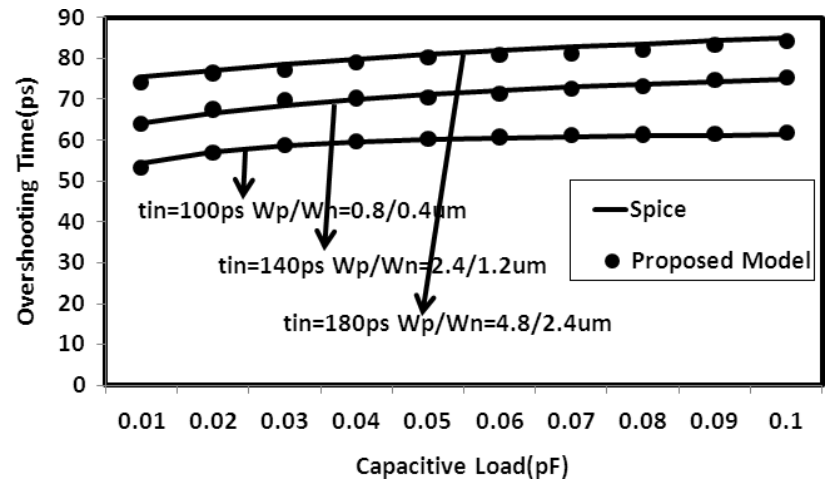

Fig. 14. The overshooting time $t_{\mathrm{ov}}$ of the proposed model and SPICE simulation for CMOS NOR gate (Fig. 4) with respect to various capacitive loads. 
$W_{N}$ varies from 80 to $800 \mathrm{~nm}$ with $W_{P}=2 W_{N}$ and $L_{P}=L_{N}=40 \mathrm{~nm}$. The load capacitance $C_{L}$ varies from 0.01 to $0.1 \mathrm{pF}$ and the length of PMOS and NMOS are $L_{P}=L_{N}=40 \mathrm{~nm}$.

Simulation results in Figs. 12-14 show that the overshooting time obtained from the proposed model is very close to the SPICE simulation results. The error of the proposed model under the first input condition is less than $3.6 \%$.

Furthermore, Figs. 15-17 show the overshooting time obtained from the proposed model and SPICE simulations for the second input condition with respect to

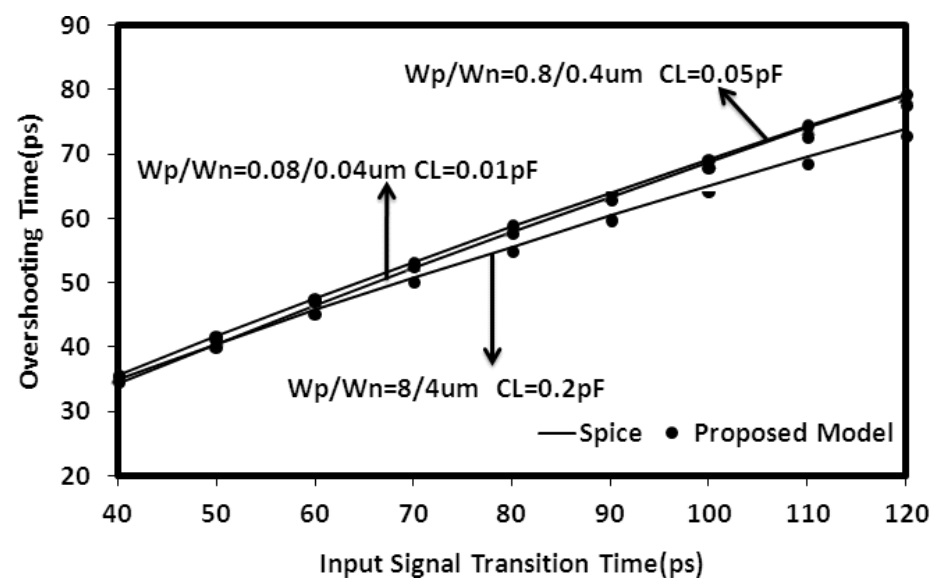

Fig. 15. The overshooting time $t_{\mathrm{ov}}$ of the proposed model and SPICE simulation for CMOS NOR gate (Fig. 7) with respect to various input signal transition times.

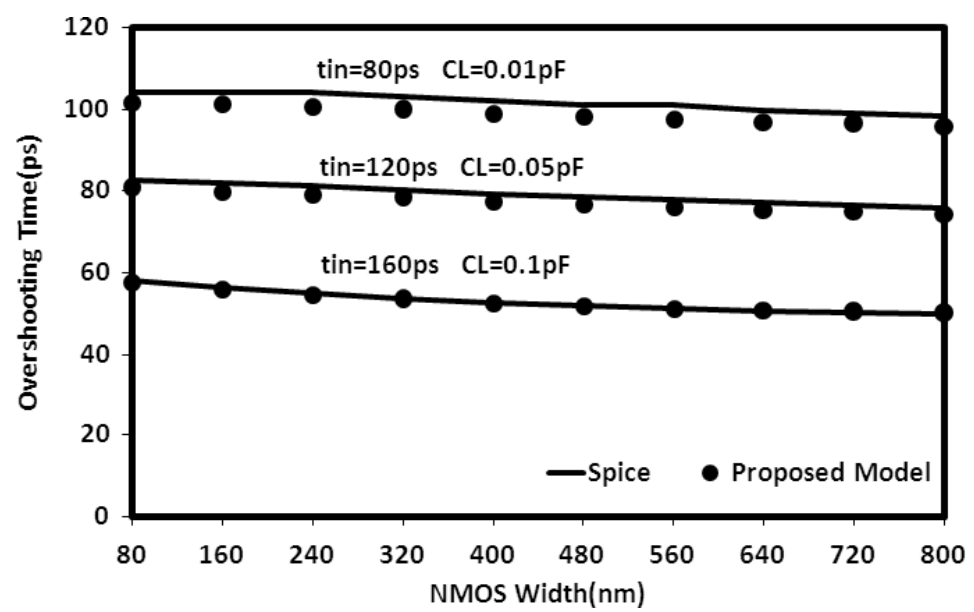

Fig. 16. The overshooting time $t_{\mathrm{ov}}$ of the proposed model and SPICE simulation for CMOS NOR gate (Fig. 7) with respect to various gate sizes. 


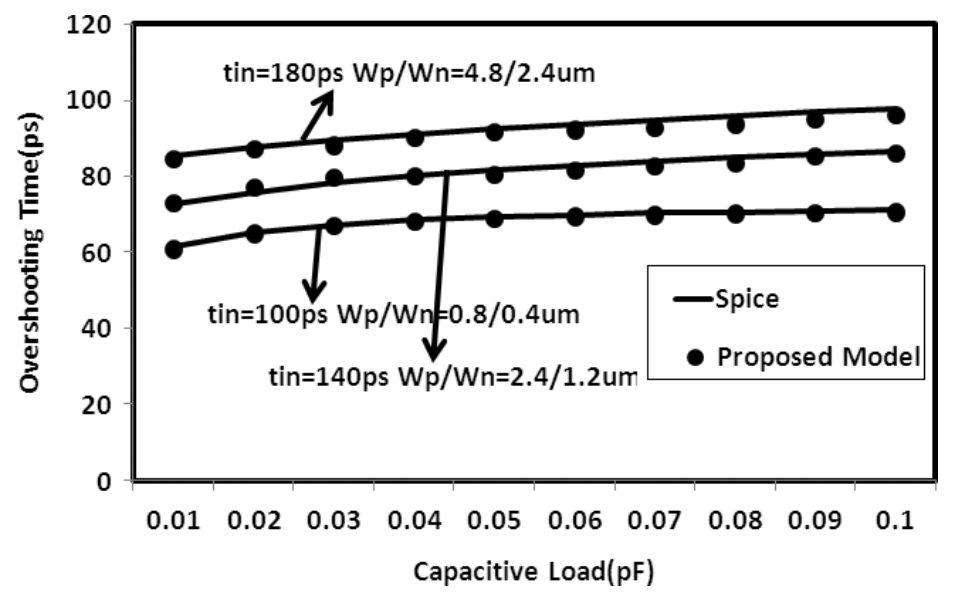

Fig. 17. The overshooting time $t_{\mathrm{ov}}$ of the proposed model and SPICE simulation for CMOS NOR gate (Fig. 7) with respect to various capacitive loads.

various input signal transition times, transistor sizes and load capacitances using 32-nm PTM model. ${ }^{11}$ All the simulation conditions are same as those of the first input condition. In addition, simulation results also show that the overshooting time obtained from the proposed model is close to the simulation results within $3.22 \%$ error.

\section{Conclusion}

With the advent of nanometer age in CMOS technology, the overshooting effect is more and more important during the analysis of gate delay. In this paper, we have modeled the overshooting effect caused in multi-input gates. The overshooting effect of CMOS NOR gate is modeled specifically. Additionally, simulation results show that the proposed model can correctly reflect the overshooting time of CMOS NOR gate compared to SPICE simulation results. Finally, the model of CMOS NOR gate considering the overshooting effect can also be easily extended to CMOS NAND gate according to the same principle.

\section{Acknowledgments}

This work is supported by Waseda University Global COE Program "International Research and Education Center for Ambient SoC" sponsored by MEXT, Japan. This work is also supported in part by VLSI Design and Education Center (VDEC), the University of Tokyo in collaboration with Synopsys, Inc. 


\section{References}

1. N. H. E. Weste and K. Eshraghian, Principles of CMOS VLSI Design: A Systems Perspective (Addison-Wesley, 1993).

2. Z. Huang, H. Yu, A. Kurokawa and Y. Inoue, Modeling the overshooting effect for CMOS inverter in nanometer technologies, IEEE Asia and South Pacific Design Automation Conf., January 2007, pp. 565-570.

3. T. Sakurai and A. R. Newton, Alpha-power-law MOSFET model and its applications to CMOS inverter delay and other formulas, IEEE J. Solid-State Circuits 25 (1990) 584-594.

4. S. Dutta, S. S. Shetti and S. L. Lushy, A comprehensive delay model for CMOS inverters, IEEE J. Solid-State Circuits 30 (1995) 864-871.

5. J. M. Daga and D. Auvergne, A comprehensive delay macro modeling for submicrometer CMOS logics, IEEE J. Solid-State Circuits 34 (1999) 42-55.

6. D. Auvergne, J. M. Daga and M. Rezzoug, Signal transition time effect on CMOS delay evaluation, IEEE Trans. Circuits Syst. I, Fundam. Theory Appl. 47 (2000) $1362-1369$.

7. A. A. Hamoui and N. C. Rumin, An analytical model for current, delay, and power analysis of submicron CMOS logic circuits, IEEE Trans. Circuits Syst. II, Analog Digit. Signal Process. 47 (2000) 999-1007.

8. P. Maurine, M. Rezzoug, N. Azemard and D. Auvergne, Transition time modeling in deep submicron CMOS, IEEE Trans. Comput.-Aided Design Integr. Circuits Syst. 21 (2002) $1352-1363$.

9. J. L. Rossello and J. Segura, An analytical charge-based compact delay model for submicron CMOS inverters, IEEE Trans. Circuits Syst. I, Fundam. Theory Appl. 51 (2004) 1301-1311.

10. Z. Huang, A. Kurokawa, M. Hashimoto, T. Sato, M. Jiang and Y. Inoue, Modeling the overshooting effect for CMOS inverter delay analysis in nanometer technologies, IEEE Trans. Comput.-Aided Design Integr. Circuits Syst. 29 (2010) 250-260.

11. Predictive Technology Model (PTM) [Online]. Available at http://ptm.asu.edu (2006). 